\title{
p.L1612P, a Novel Voltage-gated Sodium Channel Nav1.7 Mutation Inducing a Cold Sensitive Paroxysmal Extreme Pain Disorder
}

\author{
Marc R. Suter, M.D., Zahurul A. Bhuiyan, M.D., Cédric J. Laedermann, Ph.D., Thierry Kuntzer, M.D., \\ Muriel Schaller, M.D., Maurice W. Stauffacher, M.D., Eliane Roulet, M.D., Hugues Abriel, M.D., Ph.D., \\ Isabelle Decosterd, M.D., Christian Wider, M.D.
}

\begin{abstract}
Background: Mutations in the $S C N 9 A$ gene cause chronic pain and pain insensitivity syndromes. We aimed to study clinical, genetic, and electrophysiological features of paroxysmal extreme pain disorder (PEPD) caused by a novel SCN9A mutation.

Methods: Description of a 4-generation family suffering from PEPD with clinical, genetic and electrophysiological studies including patch clamp experiments assessing response to drug and temperature.

Results: The family was clinically comparable to those reported previously with the exception of a favorable effect of cold exposure and a lack of drug efficacy including with carbamazepine, a proposed treatment for PEPD. A novel p.L1612P mutation in the Nav1.7 voltage-gated sodium channel was found in the four affected family members tested. Electrophysiologically the mutation substantially depolarized the steady-state inactivation curve $\left(V_{1 / 2}\right.$ from $-61.8 \pm 4.5 \mathrm{mV}$ to $-30.9 \pm 2.2 \mathrm{mV}, \mathrm{n}=4$ and $7, P<0.001$ ), significantly increased ramp current (from $1.8 \%$ to $3.4 \%, \mathrm{n}=10$ and 12 ) and shortened recovery from inactivation (from 7.2 $\pm 5.6 \mathrm{~ms}$ to $2.2 \pm 1.5 \mathrm{~ms}, \mathrm{n}=11$ and 10 ). However, there was no persistent current. Cold exposure reduced peak current and prolonged recovery from inactivation in wild-type and mutated channels. Amitriptyline only slightly corrected the steady-state inactivation shift of the mutated channel, which is consistent with the lack of clinical benefit.

Conclusions: The novel p.L1612P Nav1.7 mutation expands the PEPD spectrum with a unique combination of clinical symptoms and electrophysiological properties. Symptoms are partially responsive to temperature but not to drug therapy. In vitro trials of sodium channel blockers or temperature dependence might help predict treatment efficacy in PEPD.

(ANESTHEsiology 2015; 122:414-23)
\end{abstract}

$\mathbf{P}$ AROXYSMAL extreme pain disorder (PEPD) is a chronic, genetically determined disease, characterized by episodes of excruciating pain and flushing upon benign mechanical or heat stimuli mostly in rectal, ocular or submandibular regions. ${ }^{1-4}$ Additional symptoms include extension of pain to the lower limbs, bradycardia, tonic nonepileptic seizures and syncope. PEPD is caused by heterozygous mutations in the sodium channel, voltage-gated type $I X$, $\alpha$-subunit gene (SCN9A) which encodes the Nav1.7 sodium channel. ${ }^{4}$ Disease-causing mutations lead to a channel gainof-function through depolarizing shift of voltage-dependence of inactivation of Nav1.7 sometimes associated with persistent current, which increases excitability of sensory neurons.

\section{What We Already Know about This Topic}

- Mutations of the sodium ion channel SCN9A gene have been associated with syndromes of both heightened and diminished pain sensitivity

- Those mutations supporting channel over activity and heightened pain sensitivity often respond to sodium ion channel blocking molecules

\section{What This Article Tells Us That Is New}

- A new mutation, p.L1612P, was found in four family members suffering from Paroxysmal Extreme Pain Disorder

- The mutant ion channel possesses unique electrophysiological characteristics, and a pharmacological profile distinct from other known mutations

This work has been presented at the European Federation of International Association for the Study of Pain chapters (EFIC) congress, Florence, Italy, October 11, 2013, and the Swiss Society for the Study of Pain, Biel, Switzerland, December 12, 2013 (Suter MR, Schaller M, Decosterd I, Wider C: L1612P, a new paroxysmal extreme pain disorder-causing Nav1.7 mutation shows unique combination of electrophysiological properties); and the Swiss Society of Anesthesiology, Lausanne, Switzerland, November 8, 2013 (Suter MR, Schaller M, Stauffacher M, Bhuiyan ZA, Decosterd I, Wider C: Expanding the genetic and electrophysiological spectrum of Paroxysmal extreme pain disorder (PEPD): A novel L1612P Nav1.7 mutation study). Drs. Decosterd and Wider contributed equally to this work.

Submitted for publication June 25, 2014. Accepted for publication September 4, 2014. From the Pain Center, Department of Anesthesiology, Lausanne University Hospital (CHUV) and University of Lausanne, Lausanne, Switzerland (M.R.S., C.J.L., I.D.); Service of Medical Genetics, Department of Laboratories, Lausanne University Hospital (CHUV), Lausanne, Switzerland (Z.A.B.); Faculty of Biology and Medicine, University of Lausanne, Lausanne, Switzerland (T.K., M.S., M.W.S., C.W.); Department of Clinical Neurosciences, Lausanne University Hospital (CHUV), Lausanne, Switzerland (T.K., C.W.); Department of Pediatrics, Lausanne University Hospital (CHUV), Lausanne, Switzerland (E.R.); Department of Clinical Research, University of Bern, Bern, Switzerland (H.A.); and Department of Fundamental Neurosciences, University of Lausanne, Lausanne, Switzerland (I.D.). 
Less than 20 families with PEPD have been reported worldwide. ${ }^{5,6}$ Other mutations in SCN9A cause disorders such as congenital insensitivity to pain, erythromelalgia, and smallfiber neuropathy and participate in painful not paroxysmal pain syndromes. ${ }^{7,8}$ Inherited erythromelalgia (IEM) inducing mutations also increase excitability of sensory neurons but classically through a hyperpolarizing shift of the activation. Typical symptoms of affected patients are peripheral, bilateral severe burning pain of affected extremities associated with erythema, triggered by warmth, stress and exhaustion and often relieved by cooling of the affected sites. ${ }^{9}$ Nonpharmacological measures to prevent or treat painful attacks such as immersion in ice-cold water were mostly described in patients suffering from erythromelalgia. ${ }^{10}$ The therapeutic effect of cold is not described in PEPD. The two diseases therefore present with distinct electrophysiological and clinical hallmarks. One p.A1632E mutation combines the stereotypical characteristics of both diseases with electrophysiological demonstration of the depolarizing shift of steady-state inactivation as well as the hyperpolarizing shift of activation seen in PEPD and IEM, respectively. Cooling improves the patient's symptoms. ${ }^{11}$

The sodium channel blocker carbamazepine is the treatment of choice for PEPD; ${ }^{4}$ however it fails to alleviate pain in some patients. Therefore, other therapeutic options need to be investigated and the electrophysiological and clinical features of each individual mutation should be taken into account.

Amitriptyline which is a first line drug for neuropathic pain ${ }^{12}$ blocks the Nav1.7 sodium channel. ${ }^{13}$ Other sodium channel blockers such as lidocaine have been used successfully in neuropathic pain of different etiologies ${ }^{14-17}$ including Nav1.7 mutations. ${ }^{18}$ However, administration of lidocaine requires an intravenous line and despite some patients reporting prolonged improvement after the infusion, ${ }^{16}$ an orally available sodium channel blocker is preferable for long term therapy. Mexiletine, the oral drug usually used after positive intravenous lidocaine trials, ${ }^{18,19}$ is not available in many countries and is only marketed for cardiac conditions. Amitriptyline is sometimes used as an alternative following lidocaine. ${ }^{20}$ Herein, we report detailed clinical and genetic investigation of a family with PEPD caused by a novel mutation in the $S C N 9 A$ gene; in vitro electrophysiological evidence of pathogenicity; and effects of amitriptyline and cold exposure both at the clinical and electrophysiological levels.

\section{Material and Methods}

\section{Clinical and Genealogical Investigation}

The family originates from Portugal, with several members now living in Switzerland, Spain, and Australia. Partial clinical data on selected members were previously published (Family 13 in Fertleman et al.). ${ }^{6}$ Patients and at-risk family members were reinvestigated by means of a semistructured interview, either in person or over the phone. Data collected included demographic information, as well as details on the nature, distribution, and triggering factors of symptoms. Those affected individuals who were available underwent thorough neurological examination (Thierry Kuntzer, Christian Wider, Marc R. Suter); nerve conduction studies were performed on the index patient. The study was accepted by the institutional review board of the Canton de Vaud, Lausanne, Switzerland. All individuals enrolled provided written informed consent.

\section{Genetic Study}

DNA was extracted from lymphocytes using standard methods. Samples from all four available affected individuals underwent sequencing of all exons and exon-intron boundaries of the SCN9A gene. Methods employed included polymerase chain reaction amplification and Sanger sequencing (primers available upon request). Following identification of the novel SCN9A p.L1612P mutation, samples from all available family members were genotyped using the same methods. Ninety-two Swiss adults seen at the genetics service for other disorders without any history of chronic pain were also assessed for presence of the p.L1612P mutation.

\section{Plasmid and Cell Line}

The p.L1612P substitution was inserted into a plasmid containing the human Nav1.7 insert using QuikChange XL II site directed mutagenesis kit (Agilent Technologies, Basel, Switzerland). Human embryonic kidney 293 cells were cultured in Dulbecco's Modified Eagle Medium (GIBCO cat\#41965 039, Invitrogen, Merelbeke, Belgium) supplemented with $10 \%$ fetal bovine serum, L-Glutamine $4 \mathrm{mM}$ (GIBCO cat\#25030 024, Invitrogen, Merelbeke, Belgium) and gentamicin $0.02 \mathrm{mg} / \mathrm{ml}$ (Invitrogen cat\# 15750-037, Paisley, Scotland, United Kingdom). Transient transfection of either wild-type (WT) human Nav1.7 or L1612P mutant plasmid associated with three plasmids with inserts for $\beta 1$ and $\beta 2$ subunits of sodium channels and green fluorescent protein, was performed using jetPEI (Polyplus, Illkirch, France), with the ratio $\alpha: \beta 1: \beta 2$ :green fluorescent protein being 20:2:2:1 as previously described. ${ }^{21}$ One day after transfection, cells were plated to be recorded at $48 \mathrm{~h}$.

\section{In Vitro Electrophysiology}

Measurements were made using pClamp software, version 10.3, and a multiclamp 700B amplifier (Molecular Devices, LLC, Sunnyvale, CA) at room temperature, if not otherwise specified. The sampling rate was $100 \mathrm{kHz}$ with a low pass Bessel filter at $10 \mathrm{kHz}$. Data were smoothed and analyzed using Clampfit software version 10.3.1.5 (Molecular Devices, LLC), and KaleidaGraph (Synergy Software, Reading, PA).

Whole-cell Patch clamp recordings were conducted using an internal solution containing (in $\mathrm{mmol} / \mathrm{L}$ ) $\mathrm{CsCl} 60$, Cesium aspartate 70 , EGTA $11, \mathrm{MgCl}_{2} 1, \mathrm{CaCl}_{2} 1$, HEPES 10 , and $\mathrm{Na}_{2}$-adenosine triphosphate $5, \mathrm{pH}$ adjusted to 7.2 with $\mathrm{CsOH}$; and an external solution containing $\mathrm{NaCl} 130$, $\mathrm{CaCl}_{2} 2, \mathrm{MgCl}_{2} 1.2, \mathrm{CsCl} 5$, HEPES 10, and glucose 5, 
$\mathrm{pH}$ adjusted to 7.4 with $\mathrm{CsOH}$. Holding potential was $-100 \mathrm{mV}$. The values were not corrected for liquid junction potential. Pipette resistance was ranging from 2 to $7 \mathrm{MOhm}$. Once whole cell configuration was obtained, pipette and cell capacitance were minimized using the muticlamp commander. To reduce voltage errors, $80-90 \%$ series resistance compensation was applied. Only data from cells having stable access resistance over the duration of the experiment were used. Cells for which signs of poor voltage-clamp control, such as delayed inflections of the current or discontinuities in the peak $I_{\mathrm{Na}}$ versus $V_{\mathrm{m}}$ curve were not analyzed. Protocols are shown as insets in the figures. The experimenter was not blinded during the electrophysiological studies. Everything was done to avoid bias. Cell culture and transfection were performed in parallel for both genotype and cells containing the plasmid from the two genotypes were alternatively patched.

Peak currents were measured from the activation protocol. The linear ascending segment of the current-voltage relationship was used to estimate the reversal potential for each trace before converting peak current to conductance and obtaining the voltage-dependent activation curve. Voltage-dependence of activation and steady-state inactivation curves were individually fitted with Boltzmann relationships, $y\left(V_{\mathrm{m}}\right)=1 /\left(1+\exp \left[\left(V_{\mathrm{m}}-V_{1 / 2}\right) / K\right]\right)$ in which $y$ is the normalized current or conductance, $V_{\mathrm{m}}$ is the membrane potential, $V_{1 / 2}$ is the voltage at which half of the channels are activated or inactivated and $K$ is the slope factor. The decaying phase of current elicited in the activation protocol was plotted as a single exponential $(I=\mathrm{A} \exp [-t / \tau]+\mathrm{C})$. For ramp current data were reduced by a factor of 200 with clampfit and correlated to peak current of the same cell. The $t_{1 / 2}$ value of recovery from inactivation was calculated by interpolation from a linear relation between the 2 points juxtaposing half recovery $(\mathrm{y} 1<0.5<\mathrm{y} 2)$, using the relation $x=[0.5-(\mathrm{y} 1 \mathrm{x} 2-\mathrm{y} 2 \mathrm{x} 1) /$ $(\mathrm{x} 2-\mathrm{x} 1)] \times(\mathrm{x} 2-\mathrm{x} 1) /(\mathrm{y} 2-\mathrm{y} 1) \cdot{ }^{13}$ For slow inactivation the curve was fitted with a Boltzmann $y=\operatorname{Imin}+(\operatorname{Imax}-\operatorname{Imin}) / 1+$ $\exp ((\mathrm{V} 50-\mathrm{X}) /$ slope $)$.

For Drug Testing. Amitriptyline (A8404) was purchased from Sigma (Buchs, Switzerland). Cells were perfused with extracellular medium until stable recordings were obtained (predrug). Amitriptyline was then perfused until stabilization of the peak current, followed by the recording protocol detailed above.

To assess the effect of temperature cells were perfused at $15^{\circ} \mathrm{C}$ and $25^{\circ} \mathrm{C}$ using a temperature controller (Warner Instrument TC-344B, Hamden, CT). Recordings were performed whenever the stable desired temperature was obtained. Approximately $60 \%$ of the cells reaching whole cell configuration passed through the two-temperature protocol and half of these recordings could be analyzed.

\section{Statistical Analysis}

Data are presented as mean $\pm S D$ and were analyzed using two-tailed Student's $t$ test to compare $V_{1 / 2}$, peak current, and $t_{1 / 2}$ of recovery (paired for drug and temperature effect). Two-way analysis of variance was performed to examine decay curve, followed by Bonferroni's correction for multiple testing. $P<0.05$ was used as the level of significance. No power calculation was performed; our sample size was based on previous experiment. ${ }^{13}$ Statistical analysis was performed on GraphPad Prism, version 5.03 (GraphPad Software, La Jolla, CA).

\section{Results}

\section{Clinical and Genealogical Characteristics}

The family tree is presented (fig. 1A), showing seven affected individuals (two women) over four generations, with three patients examined directly, and DNA samples collected from four affected and three unaffected family members (individuals with ${ }^{*}$ in fig. 1A). Clinical characteristics are summarized in figure 1B. Briefly, symptoms began in early infancy (range: from $1^{\text {st }}$ day of life to $1 \mathrm{yr}$ ), with all patients having paroxysmal pain and flushing in the rectal/buttocks area and in the lower limbs, and two patients in the ocular and submandibular regions (III.2, IV.1). One patient also had symptoms in the upper limbs (IV.1). Triggers included light touch such as changing diapers, trauma, defecation and warm temperature. Strategies employed by the patients to shorten/abort the attacks included immersing themselves in ice-cold water. The favorable effect of cold water exposure led to major daily life adaptations such as presence of icy water bathtubs at school and a mechanical device to lift patients into the bathtub at home. In addition to pain and flushing, the index patient (III.2) described episodes when she would present with persistent weakness in the lower limbs for hours or even days after an attack, sometimes requiring hospitalization. She presented around the age of $1 \mathrm{yr}$ with episodes of loss of consciousness that prompted investigations for epileptic seizures or cardiac arrhythmia. The clinical diagnosis of PEPD was only established later in her affected son. General neurologic examination was normal; motor and sensory nerve conduction studies were normal in the median and ulnar nerves in the index patient, and latency measurement of myoelectric deep tendon ( $\mathrm{T}$ ) reflex responses from soleus and rectus femoris muscles was also normal ${ }^{22}$ (the patient did not tolerate electrical shocks in the lower limbs for fear of triggering a painful attack). Carbamazepine was tried in two patients (III.2, IV.1). Patient III.2 reached $1,200 \mathrm{mg} / \mathrm{d}$ of carbamazepine but suffered from side effect (loss of balance, impairment in language and vision). Only minor benefit was found in patient IV.1. Amitriptyline and pregabalin proved ineffective in the index patient.

\section{Genetic Study}

Sequencing of the $S C N 9 A$ gene identified a novel c. $4835 \mathrm{~T}>\mathrm{C}$ (p.L1612P) mutation in all four available affected individuals but not in the three at-risk unaffected family members, indicating perfect cosegregation with disease. In addition, 


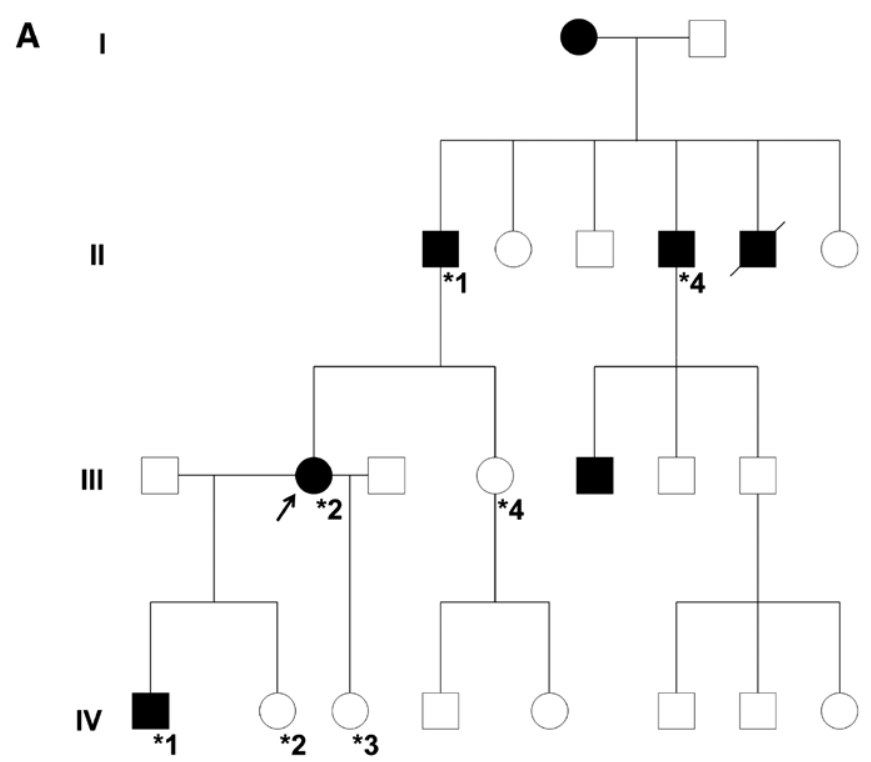

B

\begin{tabular}{|c|c|c|c|c|c|c|c|}
\hline $\begin{array}{c}\text { Pedigree } \\
\text { number }\end{array}$ & Gender & $\begin{array}{c}\text { Age } \\
\text { (years) }\end{array}$ & $\begin{array}{c}\text { Onset } \\
\text { age }\end{array}$ & $\begin{array}{c}\text { SCN9A } \\
\text { p.L1612P }\end{array}$ & Symptoms & Treatments & $\begin{array}{c}\text { Treatment } \\
\text { effect }\end{array}$ \\
\hline II.1 & M & 60 & 1 day & + & R/LL/F & none & NA \\
\hline II.4 ${ }^{\star}$ & M & U & U & + & U & U & U \\
\hline III.2 & F & 36 & $\sim 1$ year & + & R/LL/F/O/SM/ & CBZ, PGB, & Partial (PPX \\
\hline III.4 & F & 31 & NA & - & none & NA & NA R \\
\hline IV.1 & M & 11 & $\sim 1.5$ & + & R/LL/F/O/UL/ & CBZ & Minor \\
\hline IV.2 & F & 15 & NA & - & none & NA & NA \\
\hline IV.3 & F & 10 & NA & - & none & NA & NA \\
\hline
\end{tabular}

Fig. 1. Pedigree and clinical data. $(A)$ Index patient (arrow) and pedigree of her family: *DNA sample available; squares: males; circles: females; black: affected; white: unaffected. $(B)$ Clinical characteristics of family members available for the genetic study. + = present; - = absent; AMI = amitriptyline; CBZ = carbamazepine; F = flushing; $L L$ = lower limbs; NA = not applicable; $\mathrm{O}=$ ocular; $\mathrm{PGB}=$ pregabalin; $\mathrm{PPX}=$ pramipexole; $\mathrm{R}=$ rectal/buttocks; $\mathrm{RL}=$ restless-legs symptoms; $\mathrm{SM}=\mathrm{submandibular} ;$ $\mathrm{U}=$ unavailable; $\mathrm{UL}=$ upper limbs. *No details were available for patient II.4 who only donated a blood sample.

the c.4835T>C mutation was not found in 92 healthy controls of Swiss descent. Further supporting pathogenicity is the fact that the non-synonymous mutation lies in close vicinity to other previously reported pathogenic SCN9A mutations, within a region highly conserved across species.

\section{In Vitro Study of the Mutated Channel}

p.L1612P Displays Slower Inactivation Kinetics. We analyzed the decay of sodium current (entry into the fast inactivated state) with a single exponential plotted from the current peak to the end of a $100 \mathrm{~ms}$ pulse at voltage steps from 0 to $+30 \mathrm{mV}$. An example is shown in figure $2 \mathrm{~A}$ and average time constant of decrease $(\tau)$ is shown against voltage in figure $2 \mathrm{~B}$. We observed a slower decay of current in the p.L1612P mutant compared to the WT. Decay rates were $1.28 \pm 0.63$ (WT) and $3.27 \pm 0.70 \mathrm{~ms}$ (mutant)
$(P=0.00014, \mathrm{n}=6$ for WT and $\mathrm{n}=8$ for mutant $)$ after a single depolarization at $10 \mathrm{mV}$. Mean residual current was measured 20, 50, and $100 \mathrm{~ms}$ after the peak. Figure $2 \mathrm{C}$ shows the percentage of peak current at each time-point with a significant increase between WT and mutant channels $20 \mathrm{~ms}$ after the peak. Of note, at 50 and $100 \mathrm{~ms}$ the persistent current recorded in cells expressing in WT and p.L1612P channels were not different and of very small value.

p.L1612P Shifts Voltage-Dependence of Activation and Steady-State Inactivation. Activation curves were obtained using $10 \mathrm{~ms}$ steps to potentials from -80 to $+15 \mathrm{mV}$ in 5 $\mathrm{mV}$ increments from the $-100 \mathrm{mV}$ holding potential. We observed a depolarizing shift in the activation curve of the mutant with a displacement of $V_{1 / 2}$ from $-9.0 \pm 7.2$ to $0.0 \pm 1.9 \mathrm{mV}(P=0.028, \mathrm{n}=13$ for WT and $\mathrm{n}=11$ for p.L1612P, fig. 3A). Mean peak current density measured 

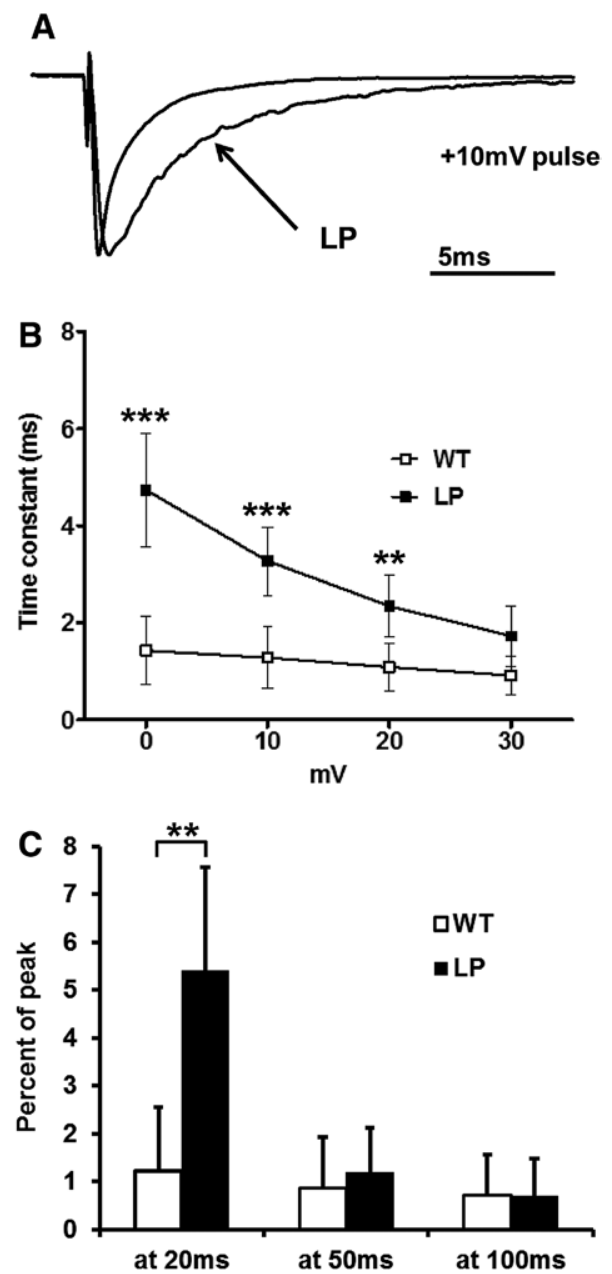

Fig. 2. Current characteristics of WT and mutant channels. (A) Recordings of both WT and p.L1612P mutant channels, normalized to the same peak current. $(B)$ Slower current decay of the p.L1612P channel. (C) No difference in persistent current is observed between the WT and p.L1612P channel at 50 and $100 \mathrm{~ms}$ and its value reaches below $1 \%$ at $100 \mathrm{~ms}$ after the peak. LP $=$ p.L1612P; $\mathrm{WT}=$ wild type ${ }^{* \star} P<0.01$ and $\left.{ }^{\star \star \star} P<0.001\right)$.

from the current-voltage protocol were not different $(316.2 \pm 266.9 \mathrm{pA} / \mathrm{pF}$ for $\mathrm{WT}$ and $274.5 \pm 278.7 \mathrm{pA} / \mathrm{pF}$ for p.L1612P, $\mathrm{n}=15$ and $19, P=0.67)$. For steady-state fast inactivation curve (SSI) $500 \mathrm{~ms}$ prepulses ranging from -130 to $+5 \mathrm{mV}$ followed by a $20 \mathrm{~ms}$ pulse to $0 \mathrm{mV}$ were applied. The p.L1612P substitution largely shifted SSI to more depolarized values with a difference in $V_{1 / 2}$ of $30.9 \mathrm{mV}$ $(-61.8 \pm 4.5$ for WT and $-30.9 \pm 2.2 \mathrm{mV}$ for p.L1612P, $\mathrm{n}=$ 4 and $7, P<0.001$, fig. 3A). The p.L1612P mutant showed full inactivation at the end of the SSI protocol.

Recovery from Inactivation Is Hastened by the p.L1612P Mutation. To measure recovery from fast inactivation, we used a double pulse protocol with varying interpulse interval and plotted the ratio of the second to the first pulse to the interval. The $t_{1 / 2}$ of recovery from inactivation was significantly faster in cells expressing the mutant channels
$(2.2 \pm 1.5$ and $7.2 \pm 5.6 \mathrm{~ms}$ for p.L1612P and WT, $\mathrm{n}=10$ and $11, P=0.013$, fig. 3B).

The p.L1612P Mutation Increases Ramp Current. To assess the theoretical increase in window current seen when superposing activation and SSI curves, we looked at the response of WT and mutant channels to slow ramp depolarization from -100 to $+20 \mathrm{mV}$ at a rate of 0.2 $\mathrm{mV} / \mathrm{ms}$. Ramp current was normalized to the peak current. We observed a significant difference $(P=0.0018)$ in maximum ramp current between WT $(1.8 \pm 1.4 \%, \mathrm{n}=$ $10)$ and p.L1612P channels $(3.4 \pm 0.7 \%, \mathrm{n}=12)$ (fig. $3 \mathrm{C})$. The peaks of ramp current were at $-7.4[-37.2$ to +1.2$]$ and $-8.4[-12$ to +2$] \mathrm{mV}$ (median [range]) for WT and mutant channels $(P=0.2)$.

The p.L1612P Substitution Does Not Alter Voltage-dependence of Slow Inactivation. To study voltage-dependence of slow inactivation, we used a $30 \mathrm{~s}$ duration prepulse at voltages ranging from -120 to $+30 \mathrm{mV}$ followed by a short $100 \mathrm{~ms}$ pulse to $-120 \mathrm{mV}$ in order to let the channels recover from fast inactivation and a $50 \mathrm{~ms}$ test pulse to $0 \mathrm{mV}$ to activate the available channels. We did not observe any difference in $V_{1 / 2}$ of slow inactivation $(-39.6 \pm 3.3$ and $-35.7 \pm 7.4$ $\mathrm{mV}, \mathrm{n}=8$ and 12 for WT and p.L1612P, $P=0.19$, fig. 3D) and no difference in the fraction of current resistant to slow inactivation $(29 \pm 7 \%$ vs. $33 \pm 9 \%, P=0.27)$.

Amitriptyline Affects WT and p.L1612P. Amitriptyline at a concentration of $10 \mu \mathrm{M}$ induced a similar reduction in peak current in WT and mutant channels $(33.9 \pm 8.5 \%$ and $30.0 \pm 7.6 \%$, normalized traces in fig. $4 \mathrm{~A})$. Amitriptyline did not influence the entry into the fast inactivated state $(P=0.36$ and $0.33, \mathrm{n}=5$ and 4 , for the treatment factor in two-way analysis of variance for WT and p.L1612P, fig. 4B). Amitriptyline had no effect on activation curves of both WT and p.L1612P channels (fig. 4C). Amitriptyline shifted SSI to more hyperpolarized values in WT (-7.96 $\mathrm{mV}$ ) and mutant channels (-8.51 mV) (fig. 4D). Amitriptyline inhibited the recovery from fast inactivation, prolonging $t_{1 / 2}$ of recovery (fig. 4E). Amitriptyline did reduce peak ramp current in WT but did not reach significance in p.L1612P (fig. 4F).

Both p.L1612P and WT Currents Are Altered by Cold Temperature. Cooling the recorded cells from $25^{\circ}$ to $15^{\circ} \mathrm{C}$ reduced peak current density for both WT and p.L1612P mutants (respectively $45.4 \%$ and $35.9 \%$, fig. 5, A and B), and slowed current decay over a large voltage range (fig. 5C). Voltage-dependence of activation curves was shifted to more depolarized potentials (fig. 5D). $V_{1 / 2}$ of SSI was not influenced by the change in temperature (fig. $5 \mathrm{E}$ ). Recovery from inactivation was slowed at $15^{\circ}$ compared to $25^{\circ} \mathrm{C}$ (fig. $5 \mathrm{~F}$ ). The ramp current was slightly increased for WT but not for the mutant channel.

\section{Discussion}

We report a novel p.L1612P mutation in the $S C N 9 A$ gene in a family with PEPD. Overall, clinical features were comparable 

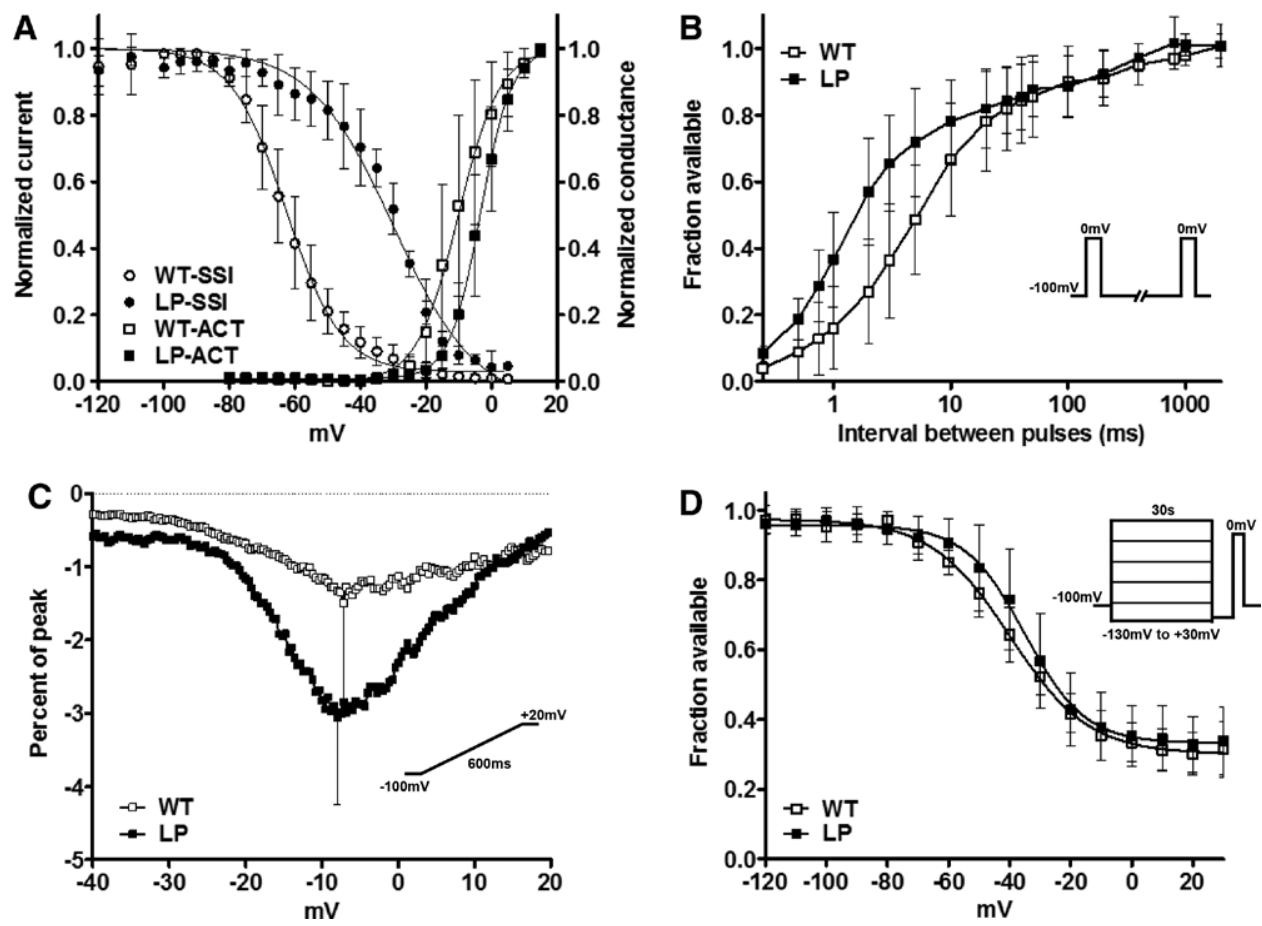

Fig. 3. Electrophysiological properties of WT and mutant channels. $(A)$ The p.L1612P mutation significantly shifts activation and SSI properties towards depolarization; the amplitude of shift is much greater for the SSI. The mutant p.L1612P channel displays shorter recovery from inactivation $(B)$ and increased ramp current $(C)$ but no difference in $V_{1 / 2}$ of slow inactivation $(D)$. ACT $=$ activation; LP = p.L1612P; SSI = steady-state inactivation; WT = wild type. Stimulation protocols are shown as insets.

to those reported in other families suffering from PEPD with the exception of symptom relief by cold exposure, presence of pain in the upper limbs in one patient and long-lasting lower limb weakness after paroxysmal episodes of pain. ${ }^{6}$ Careful clinical examination and nerve conduction studies failed to provide an explanation for limb weakness beyond fear of pain reactivation. Clinical response to carbamazepine, amitriptyline and pregabalin was absent or limited. Genetic findings demonstrated a novel mutation in SCN9A, with conclusive genetic and in vitro evidence of pathogenicity. Electrophysiologically the p.L1612P mutant channel displayed a large depolarizing shift of the SSI curve, increased ramp current, faster recovery from inactivation, and slowing of fast inactivation without persistent current, all together suggesting a channel gain of function and cell hyperexcitability.

Voltage-gated sodium channels comprise four domains (DI-DIV) of 6 transmembrane segments (S1-S6) each, with Nav1.7 residue Leu1612 being located within the S4S5 linker of DIV. Upon membrane depolarization Nav1.7 channels are activated allowing sodium entry into the cells; they then enter into inactivated states before returning to the closed state upon repolarization of the membrane. Fast inactivation relies on the interaction between the Ile-Phe-Met (IFM) residues motif located between DIII and DIV and the S4-S5 linker of DIV. ${ }^{23,24}$ Most of the previously described PEPD-causing mutations were found to be located either within the IFM motif or in the S4-S5 linker of DIII or DIV, as is the case for p.L1612P.
The magnitude of the SSI depolarizing shift with p.L1612P channels $(30.9 \mathrm{mV})$ is larger than in previously reported mutations, with the exception of neighboring p.G1607R $(31.5 \mathrm{mV}) .^{5}$ p.L1612P is the second PEPD-causing mutation to induce a depolarizing shift of the activation curve; only p.G1607R displays a $4.3 \mathrm{mV}$ depolarizing shift (9 $\mathrm{mV}$ in the present study). ${ }^{5}$ Other PEPD-causing mutations do not affect activation properties except for the hyperpolarizing shift of p.A1632E whose carriers also exhibited IEM symptoms. ${ }^{11}$ It has been proposed that Nav1.7 is responsible for triggering action potential from subthreshold oscillations. ${ }^{25}$ This may be because of the window current, which consists of the voltage values under the intersection of activation and SSI curves where a portion of channels remain open at steady-state. The window current in p.L1612P mutants is increased by the SSI depolarizing shift despite activation shift. Experimentally window current is observed through the response to a slow ramp stimulation which is increased here. Mutation in the IFM motif displays ramp currents of 4\% (p.I1461T), DIII S4-S5 mutations of 5 and 6\% (p.V1298F and p.V1299F) and DIV S4-S5 linker mutations of 4.2 and $7.0 \%$ (p.A1632E and p.M1627K). ${ }^{11,24,26}$ Interestingly, the neighboring p.G1607R mutation shows a much wider ramp current of $15.7 \%$ (ramp speed of 0.24 compared to our $0.2 \mathrm{mV} / \mathrm{ms}$ ). ${ }^{5}$ Response to ramp stimulation has occasionally been reported to include two peaks depending on the rate of ramp or on persistent current. ${ }^{27}$ Our ramp current corresponds to the rate of ramp because 
A
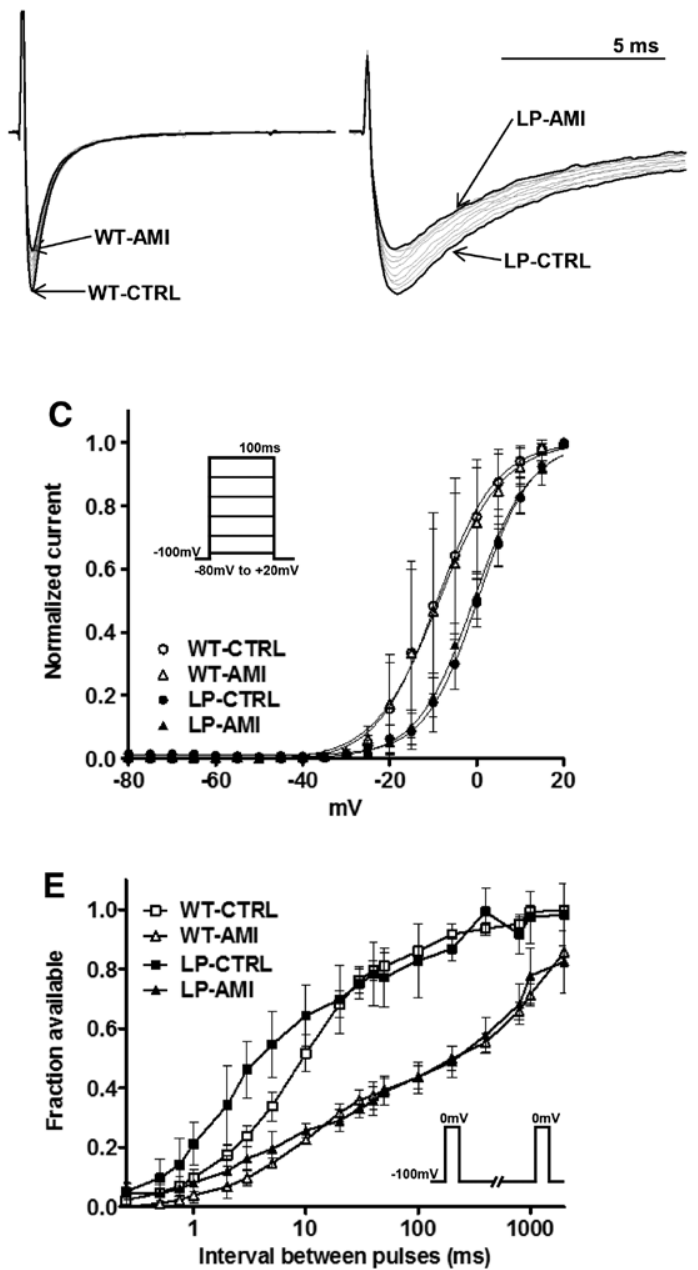
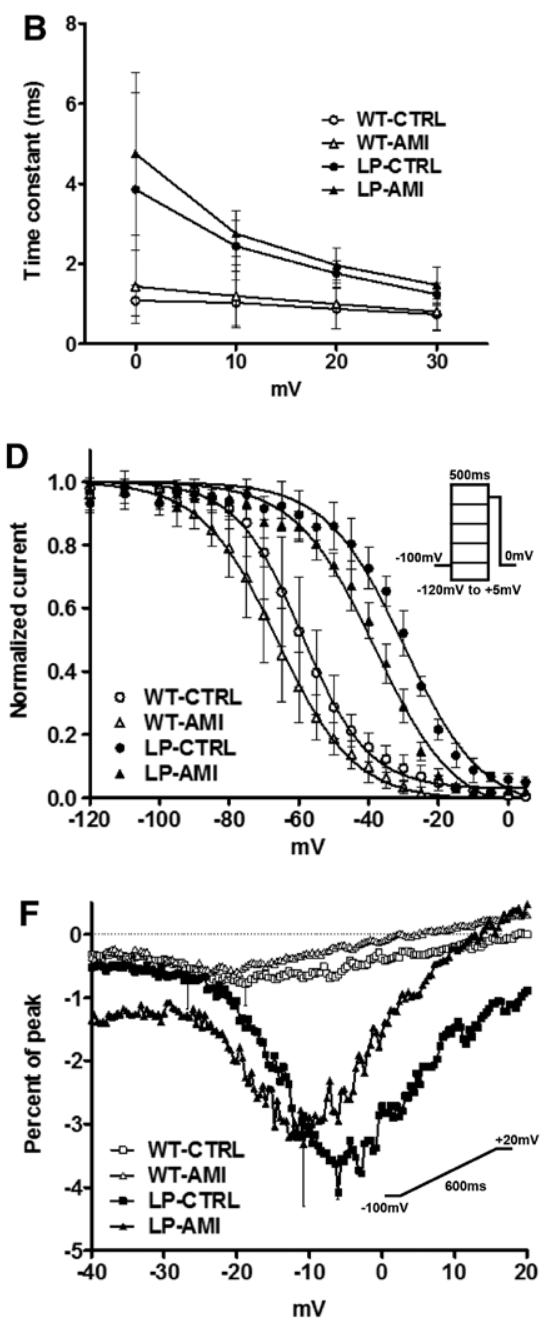

Fig. 4. Effect of amitriptyline on channel properties. (A) Example of Na current induced by $0 \mathrm{mV}$ depolarization at $1 \mathrm{~Hz}$ frequency from a $-100 \mathrm{mV}$ holding potential while changing amitriptyline concentration from 0 to $10 \mu \mathrm{M}$ for both WT and p.L1612P channels (normalized traces). (B) Amitriptyline does not modify the pace of inactivation. (C) Amitriptyline does not change activation properties. (D) Amitriptyline shifts SSI towards more hyperpolarized values without fully counteracting mutation-induced depolarizing shift $\left(V_{1 / 2}-58.43 \pm 5.97\right.$ to $-66.39 \pm 5.01 \mathrm{mV}, P=0.0011, \mathrm{n}=5$ for WT and $-31.16 \pm 2.53$ to $-39.67 \pm 1.76 \mathrm{mV}, P=0.0015, \mathrm{n}=4$ for p.L1612P). (E) Amitriptyline prolongs recovery from inactivation from $10.0 \pm 2.5$ to $228.1 \pm 90.4 \mathrm{~ms}$ for WT $(P=0.018, \mathrm{n}=4)$ and from $4.7 \pm 2.3$ to $247.3 \pm 108.1 \mathrm{~ms}$ for $\mathrm{p} . \mathrm{L} 1612 \mathrm{P}$ mutant channels $(P=0.021, \mathrm{n}=4)$. ( $(F)$ Amitriptyline decreased ramp current decrease of WT channel but not of p.L1612P (from $1.05 \pm 0.31$ to $0.78 \pm 0.34 \%, P=0.047, \mathrm{n}=5$ for WT and from $4.08 \pm 0.11$ to $3.32 \pm 0.98 \%, P=0.341, \mathrm{n}=3$ for p.L1612P). AMI = amitriptyline; CTRL = control; LP = p.L1612P; WT = wild type. Stimulation protocols are shown as insets.

it increased to $19.2 \%$ for p.L1612P when stimulation rate was faster $(1.2 \mathrm{mV} / \mathrm{ms})$ (data not shown) and because no persistent current was observed.

Slowing of fast inactivation, reflected as a slower current decay, was reported in all previously examined PEPD-causing mutations; however lack of persistent current appears to be a particular feature of the p.L1612P mutant. Fertleman $e t$ al. measured a persistent current even $100 \mathrm{~ms}$ after depolarization in both p.I1461T and p.T1464I mutations. ${ }^{4}$ Applying $25 \mathrm{~ms}$ depolarizing pulses, Jarecki et al. found a $15 \%$ and $8 \%$ persistent current for $\mathrm{p} . \mathrm{V} 1299 \mathrm{~F}$ and p.I1461T. ${ }^{26}$

The p.L1612P mutant channel shows complete fast inactivation, which contrasts with p.I1461T and p.T1464I displaying maximal inactivation levels of $54.7 \%$ and 63.5\%. ${ }^{4}$ This probably reflects the fact that both p.I1461T and p.T1464I affect the inactivation gate and are associated with more prominent persistent current. In mutations located within the S4-S5 linker of DIII, close to p.L1612P substitution of the present study, there was a $1.9 \%$ fraction of current remaining for p.M1627K, 6.3\% for p.G1607R and about $4 \%$ for p.A1632E (value approximated from figure). ${ }^{4,5,11}$ Faster recovery from inactivation is reported in most PEPD mutations including p.L1612P. The effect on slow inactivation is comparable to that of the p.M1627K mutation, with no major change in $V_{1 / 2}$ but a steeper voltage-dependence. ${ }^{26}$ Using the same stimulation protocol but 
A
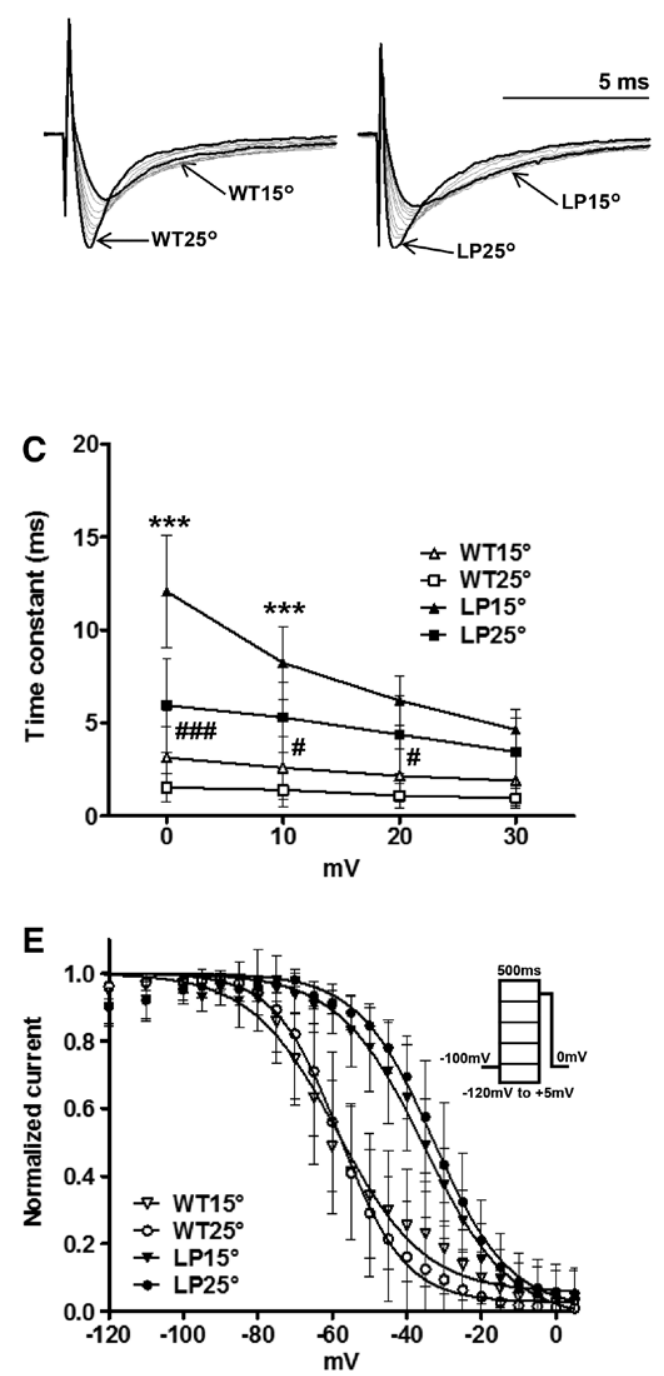
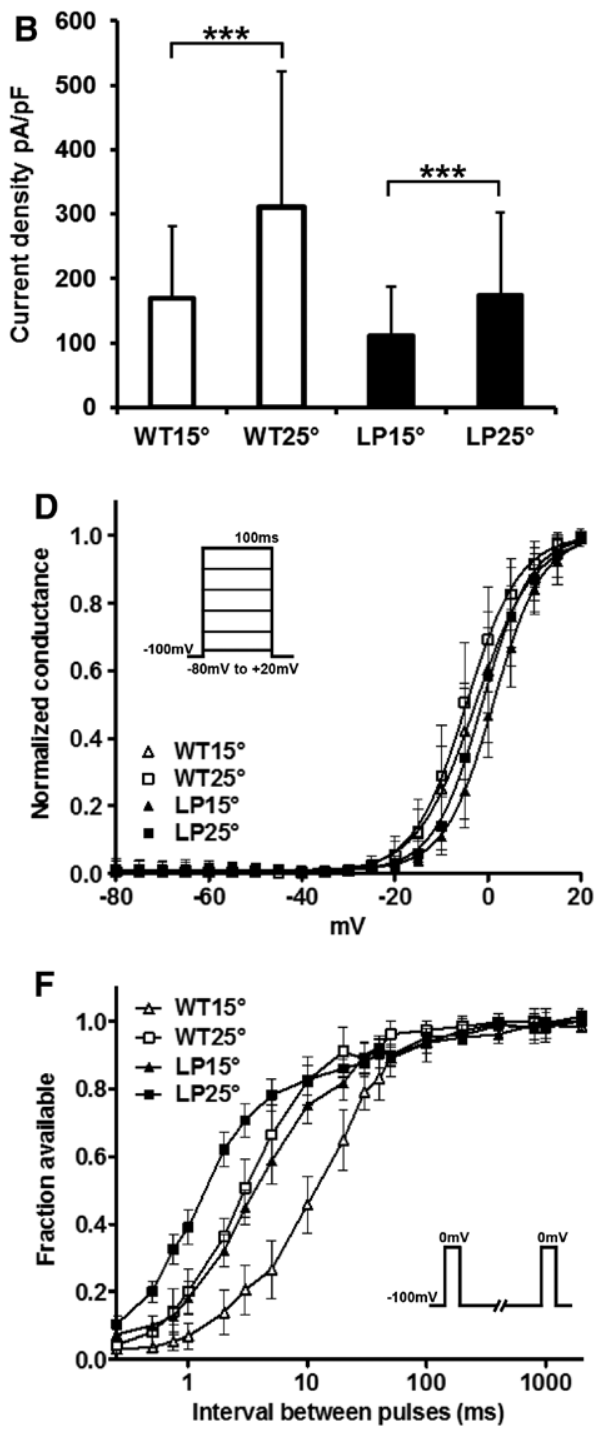

Fig. 5. Effect of temperature changes on channel properties. (A) Example of $\mathrm{Na}$ current induced by $0 \mathrm{mV}$ depolarization at $1 \mathrm{~Hz}$ frequency from a $-100 \mathrm{mV}$ holding potential while changing temperature from $15^{\circ}$ to $25^{\circ}$ for both $\mathrm{WT}$ and $\mathrm{p} \cdot \mathrm{L} 1612 \mathrm{P}$ channels (normalized traces). (B) Temperature decrease reduces mean current density in both WT and p.L1612P ${ }^{* \star *} P<0.001, n=21$ and

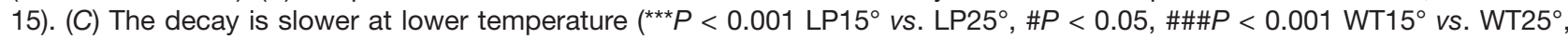
$\mathrm{n}=19$ for WT and $\mathrm{n}=14$ for p.L1612P). (D) Cooling induces a small but significant shift of $V_{1 / 2}$ of activation towards more hyperpolarized values (WT: $V_{1 / 2}$ from $-4.64 \pm 4.07$ to $-2.73 \pm 3.48 \mathrm{mV}, P=0.002, \mathrm{n}=20$; p.L1612P: $V_{1 / 2}$ from $-1.24 \pm 4.11$ to $1.09 \pm 2.57$ $\mathrm{mV}, P=0.002, \mathrm{n}=15)$. (E) No difference is seen in $V_{1 / 2}$ of inactivation between $15^{\circ}$ and $25^{\circ}$ (WT: $V_{1 / 2}$ from $-55.52 \pm 10.07$ to $-55.42 \pm 9.63 \mathrm{mV}, P=0.96, \mathrm{n}=12$; p.L1612P: $V_{1 / 2}$ from $-32.16 \pm 5.45$ to $\left.-36.19 \pm 5.99 \mathrm{mV}, P=0.12, \mathrm{n}=7\right)$. $(F)$ At $15^{\circ}$ the recovery from inactivation is slower with an increase in $t_{1 / 2}$ from $3.18 \pm 0.84$ to $12.68 \pm 3.94 \mathrm{~ms}$ for WT $(P=0.004, \mathrm{n}=5)$ and from $1.48 \pm 0.21$ to $3.86 \pm 0.88 \mathrm{~ms}$ for $\mathrm{p} . \mathrm{L} 1612 \mathrm{P}(P=0.001, \mathrm{n}=6)$. The recovery of the mutant at $15^{\circ}$ comes to the same value as for WT at $25^{\circ} . \mathrm{LP}=\mathrm{p} . \mathrm{L} 1612 \mathrm{P} ; \mathrm{WT}=$ wild type. Stimulation protocols are shown as insets.

a different intracellular patch solution, Estacion et al. showed a larger proportion of channels being resistant to inactivation in p.A1632E compared to WT channels. ${ }^{11}$ Jarecki et al. also showed alteration of slow inactivation for p.V1298F, p.V1299F and p.I1461T mostly because of the presence of two different kinetics in WT channels. ${ }^{24}$ Slow-inactivation properties are also altered in some $S C N 9 A$ mutations present in patients suffering from idiopathic small fiber neuropathy. ${ }^{28}$ In the $1739 \mathrm{~N}$ and $\mathrm{I} 720 \mathrm{~K}$ mutations, slow inactivation is modified without impairment of activation and fast-inactivation properties. ${ }^{7,28}$ Clinical features in idiopathic small fiber neuropathy patients vary and are different from the prototypical characteristics of PEPD and IEM patients, indicating that the clinical expression of a mutation not only reflects its electrophysiological properties but also other factors, such as epigenetic or environmental changes. ${ }^{7,28}$

Amitriptyline is a state-dependent sodium channel blocker which receptor overlaps with the local anesthetic receptor that is known to stabilize the inactivated state. ${ }^{29}$ Overall, the effects of amitriptyline were similar in mutant 
and WT channels and consistent with previous findings. ${ }^{13}$ Of note, the hyperpolarizing SSI shift induced by amitriptyline was far from being large enough to compensate for the depolarizing shift induced by the mutation, hallmark of electrophysiological change in PEPD inducing mutant, which might explain the lack of clinical benefit. Sodium channel blockers are used to treat chronic pain syndromes and there have been attempt to correlate in vitro effect of drugs to their clinical efficacy. ${ }^{19,30}$ Carbamazepine reduced persistent current and was efficient clinically in persistent current harboring PEPD-causing mutations. In our case, the lack of persistent current can correlate to inefficiency of carbamazepine. Carbamazepine was said to be ineffective in erythromelalgia, ${ }^{6}$ but meanwhile patients harboring p.V400M mutation were treated efficiently with this drug. Surprisingly the electrophysiological properties of this mutation do not show persistent current, but the expected hyperpolarizing shift in activation curve associated to an unusual depolarizing shift of inactivation for IEM. Carbamazepine selectively shifts the SSI and activation curves from the mutant towards the WT values which could explain its effect. ${ }^{31}$ The shift in SSI of much higher amplitude of the current p.L1612P mutation could explain the lack of efficacy in our patient. Carbamazepine also was efficient in IEM in patient harboring I848T mutation, ${ }^{32}$ which again displays a depolarizing shift in SSI besides the hyperpolarizing activation shift. ${ }^{30}$

As no drug relieved pain but cooling improved symptoms in the affected members of the described family, we investigated the effect of temperature in vitro. This has not been extensively assessed in Nav1.7 channels with PEPDcausing mutations because relief through cooling is characteristically only seen in IEM. One previous report on the IEM causing p.L858F mutation showed that reducing temperature decreased current density, slowed deactivation, increased ramp current in both WT and mutant channels, and shifted activation $V_{1 / 2}$ of WT channel in a depolarizing direction. ${ }^{9}$ We also observed decreased current density, slower inactivation, depolarizing shift of activation and increased time to recovery, however with no change in $V_{1 / 2}$ of SSI when cooling from $25^{\circ}$ to $15^{\circ}$. The relevance of the amplitude of the depolarizing shift in activation of respectively, 1.91 , and $2.33 \mathrm{mV}$ for WT and p.L1612P channel on excitability is questionable.

One property that is regulated differentially between amitriptyline and cold is the slowing of inactivation kinetics. A slower inactivation should lead to hyperexcitability but if prolonged, it could also impede the availability for subsequent opening by delayed recovery. Cold also slowed recovery from inactivation and shifted the $15^{\circ}$ mutant channel value to the WT channel room temperature value. The normalization of this property alone cannot be sufficient to hinder pain as amitriptyline even slows it to a larger extent without relieving symptoms. The clinical benefit of cold versus amitriptyline could be explained by its combined modification of biophysical properties of Nav1.7, but cold-induced pain relief might also be related to signal transduction or conduction changes independent of Nav1.7 channel.

Increased availability of patient genotyping and in vitro electrophysiology techniques may offer an opportunity for preclinical testing of drug efficacy in patients harboring SCN9A mutations. ${ }^{19}$ Amitriptyline could not restore SSI shift and window current to the values of the WT channel and was not effective in the index patient of the study. Similarly, while efficacy of carbamazepine in PEPD is probably linked to a reduction of persistent current ${ }^{4}$ the absence of such persistent current in the p.L1612P mutant could explain the lack of efficacy of carbamazepine in this family. Cooling changes the biophysical properties of the channel differentially from the drug tested which might account for its pain relieving effect. Overall, the present study illustrates how preclinical testing may help avoid laborious drug trials in patients with genetically determined pain syndromes through selection of the best candidate drugs or physical therapy.

\section{Acknowledgments}

The authors thank Christian Kern, M.D., Department of Anesthesiology, Lausanne University Hospital (CHUV), Lausanne, Switzerland, for his continuous support. They thank Marie Pertin, Pain Center, Department of Anesthesiology, Lausanne University Hospital (CHUV), for technical assistance.

Drs. Suter and Laedermann were supported by a grant (to Dr. Decosterd) from the Swiss National Science Foundation (Special Program University Medicine, Swiss Pain Research Consortium, 33CM30-124117 (2009-2012) and 33CM30_140339 (2012-2015)), Bern, Switzerland. Dr. Suter was supported by the Swiss Society of Anesthesiology, Bern, Switzerland (2009 and 2010). Dr. Abriel was supported by the Swiss National Science Foundation grant 310030_147060, Bern, Switzerland. Dr. Wider receives research support from the Leenaards Foundation, Lausanne, Switzerland.

\section{Competing Interests}

Dr. Suter received an unrestricted medical grant from Pfizer, Zurich, Switzerland, in 2013. Dr. Kuntzer has an unrestricted educational grant from CSL-Behring AG, Bern, Switzerland, for clinician-initiated research, and performs consultancy work for Actelion Ltd, Baden, Switzerland. All other authors declare no competing interests.

\section{Correspondence}

Address correspondence to Dr. Suter: Pain Center, Department of Anesthesiology, Lausanne University Hospital (CHUV), Rue du Bugnon 46, 1011 Lausanne, Switzerland. marc.suter@chuv.ch. Information on purchasing reprints may be found at www.anesthesiology.org or on the masthead page at the beginning of this issue. Anesthesiology's articles are made freely accessible to all readers, for personal use only, 6 months from the cover date of the issue.

\section{References}

1. Schubert R, Cracco JB: Familial rectal pain: a type of reflex epilepsy? Ann Neurol 1992; 32:824-6

2. Hayden R, Grossman M: Rectal, ocular, and submaxillary pain; a familial autonomic disorder related to proctalgia fugaz: report of a family. AMA J Dis Child 1959; 97:479-82 
3. Fertleman CR, Ferrie CD: What's in a name-familial rectal pain syndrome becomes paroxysmal extreme pain disorder. J Neurol Neurosurg Psychiatry 2006; 77:1294-5

4. Fertleman CR, Baker MD, Parker KA, Moffatt S, Elmslie FV, Abrahamsen B, Ostman J, Klugbauer N, Wood JN, Gardiner RM, Rees M: SCN9A mutations in paroxysmal extreme pain disorder: Allelic variants underlie distinct channel defects and phenotypes. Neuron 2006; 52:767-74

5. Choi JS, Boralevi F, Brissaud O, Sánchez-Martín J, Te Morsche RH, Dib-Hajj SD, Drenth JP, Waxman SG: Paroxysmal extreme pain disorder: a molecular lesion of peripheral neurons. Nat Rev Neurol 2011; 7:51-5

6. Fertleman CR, Ferrie CD, Aicardi J, Bednarek NA, Eeg-Olofsson $\mathrm{O}$, Elmslie FV, Griesemer DA, Goutières F, Kirkpatrick M, Malmros IN, Pollitzer M, Rossiter M, Roulet-Perez E, Schubert R, Smith VV, Testard H, Wong V, Stephenson JB: Paroxysmal extreme pain disorder (previously familial rectal pain syndrome). Neurology 2007; 69:586-95

7. Han C, Hoeijmakers JG, Ahn HS, Zhao P, Shah P, Lauria G, Gerrits MM, te Morsche RH, Dib-Hajj SD, Drenth JP, Faber CG, Merkies IS, Waxman SG: Nav1.7-related small fiber neuropathy: Impaired slow-inactivation and DRG neuron hyperexcitability. Neurology 2012; 78:1635-43

8. Dabby R, Sadeh M, Gilad R, Lampl Y, Cohen S, Inbar S, Leshinsky-Silver E: Chronic non-paroxysmal neuropathic pain-Novel phenotype of mutation in the sodium channel SCN9A gene. J Neurol Sci 2011; 301:90-2

9. Han C, Lampert A, Rush AM, Dib-Hajj SD, Wang X, Yang Y Waxman SG: Temperature dependence of erythromelalgia mutation L858F in sodium channel Nav1.7. Mol Pain 2007; $3: 3$

10. Davis MD, O'Fallon WM, Rogers RS $3^{\text {rd }}$, Rooke TW: Natural history of erythromelalgia: presentation and outcome in 168 patients. Arch Dermatol 2000; 136:330-6

11. Estacion M, Dib-Hajj SD, Benke PJ, Te Morsche RH, Eastman EM, Macala LJ, Drenth JP, Waxman SG: NaV1.7 gain-of-function mutations as a continuum: A1632E displays physiological changes associated with erythromelalgia and paroxysmal extreme pain disorder mutations and produces symptoms of both disorders. J Neurosci 2008; 28:11079-88

12. Dworkin RH, O'Connor AB, Backonja M, Farrar JT, Finnerup NB, Jensen TS, Kalso EA, Loeser JD, Miaskowski C, Nurmikko TJ, Portenoy RK, Rice AS, Stacey BR, Treede RD, Turk DC, Wallace MS: Pharmacologic management of neuropathic pain: Evidence-based recommendations. Pain 2007; 132:237-51

13. Suter MR, Kirschmann G, Laedermann CJ, Abriel H, Decosterd I: Rufinamide attenuates mechanical allodynia in a model of neuropathic pain in the mouse and stabilizes voltage-gated sodium channel inactivated state. Anesthesiology 2013; 118:160-72

14. Kalso E, Tramèr MR, McQuay HJ, Moore RA: Systemic localanaesthetic-type drugs in chronic pain: A systematic review. Eur J Pain 1998; 2:3-14

15. Galer BS, Miller KV, Rowbotham MC: Response to intravenous lidocaine infusion differs based on clinical diagnosis and site of nervous system injury. Neurology 1993; 43:1233-5

16. Mao J, Chen LL: Systemic lidocaine for neuropathic pain relief. Pain 2000; 87:7-17
17. Challapalli V, Tremont-Lukats IW, McNicol ED, Lau J, Carr DB: Systemic administration of local anesthetic agents to relieve neuropathic pain. CochraneDatabaseSystRev 2005:CD003345

18. Nathan A, Rose JB, Guite JW, Hehir D, Milovcich K: Primary erythromelalgia in a child responding to intravenous lidocaine and oral mexiletine treatment. Pediatrics 2005; 115:e504-7

19. Wu MT, Huang PY, Yen CT, Chen CC, Lee MJ: A novel SCN9A mutation responsible for primary erythromelalgia and is resistant to the treatment of sodium channel blockers. PLoS One 2013; 8:e55212

20. Fridrich P, Colvin HP, Zizza A, Wasan AD, Lukanich J, Lirk P, Saria A, Zernig G, Hamp T, Gerner P: Phase 1A safety assessment of intravenous amitriptyline. J Pain 2007; 8:549-55

21. Lossin C, Wang DW, Rhodes TH, Vanoye CG, George AL Jr: Molecular basis of an inherited epilepsy. Neuron 2002; 34:877-84

22. Péréon Y, Nguyen The Tich S, Fournier E, Genet R, Guihéneuc P: Electrophysiological recording of deep tendon reflexes: Normative data in children and in adults. Neurophysiol Clin 2004; 34:131-9

23. Smith MR, Goldin AL: Interaction between the sodium channel inactivation linker and domain III S4-S5. Biophys J 1997; 73:1885-95

24. Jarecki BW, Sheets PL, Jackson JO $2^{\text {nd }}$, Cummins TR: Paroxysmal extreme pain disorder mutations within the D3/ S4-S5 linker of Nav1.7 cause moderate destabilization of fast inactivation. J Physiol 2008; 586(Pt 17):4137-53

25. Cummins TR, Howe JR, Waxman SG: Slow closed-state inactivation: A novel mechanism underlying ramp currents in cells expressing the hNE/PN1 sodium channel. J Neurosci 1998; 18:9607-19

26. Dib-Haji SD, Estacion M, Jarecki BW, Tyrrell L, Fischer TZ, Lawden M, Cummins TR, Waxman SG: Paroxysmal extreme pain disorder M1627K mutation in human Nav1.7 renders DRG neurons hyperexcitable. Mol Pain 2008; 4:37

27. Estacion $\mathrm{M}$, Waxman SG: The response of $\mathrm{Na}(\mathrm{V}) 1.3$ sodium channels to ramp stimuli: multiple components and mechanisms. J Neurophysiol 2013; 109:306-14

28. Faber CG, Hoeijmakers JG, Ahn HS, Cheng X, Han C, Choi JS, Estacion M, Lauria G, Vanhoutte EK, Gerrits MM, Dib-Hajj S, Drenth JP, Waxman SG, Merkies IS: Gain of function Nav1.7 mutations in idiopathic small fiber neuropathy. Ann Neurol 2012; 71:26-39

29. Wang GK, Russell C, Wang SY: State-dependent block of voltage-gated $\mathrm{Na}+$ channels by amitriptyline via the local anesthetic receptor and its implication for neuropathic pain. Pain 2004; 110:166-74

30. Yang Y, Dib-Hajj SD, Zhang J, Zhang Y, Tyrrell L, Estacion M, Waxman SG: Structural modelling and mutant cycle analysis predict pharmacoresponsiveness of a $\mathrm{Na}(\mathrm{V}) 1.7$ mutant channel. Nat Commun 2012; 3:1186

31. Fischer TZ, Gilmore ES, Estacion M, Eastman E, Taylor S, Melanson M, Dib-Hajj SD, Waxman SG: A novel Nav1.7 mutation producing carbamazepine-responsive erythromelalgia. Ann Neurol 2009; 65:733-41

32. Natkunarajah J, Atherton D, Elmslie F, Mansour S, Mortimer $\mathrm{P}$ : Treatment with carbamazepine and gabapentin of a patient with primary erythermalgia (erythromelalgia) identified to have a mutation in the SCN9A gene, encoding a voltagegated sodium channel. Clin Exp Dermatol 2009; 34:e640-2 